\title{
STUDY AND SOLID-STATE CHARACTERIZATION OF ANTIHYPERTENSIVE DRUGS
}

Sato, J. A. P.*; Ferreira, F. F.

Universidade Federal do ABC, CCNH, Santo André, SP, Brazil

*juliana.sato@ufabc.edu.br

Enalapril Maleate is an antihypertensive drug currently being administered in tablet form. It is known to exist in at least two polymorphic forms, exhibiting similar stabilities. Form I crystallizes in a monoclinic system ${ }^{1}$, space group $P 2_{1}$, while Form II has been identified as an orthorhombic crystal, with space group $P 2_{1} 2_{1} 2_{1}$. Polymorphic forms of a drug substance can have different chemical and physical properties, including melting point, chemical reactivity, apparent solubility, dissolution rates, optical and mechanical properties, vapor pressure, and density. In this work we analyzed five tablets of enalapril maleate, two samples of active pharmaceutical ingredients purchased from compounding pharmacies and three tablets: generic, similar and reference. By DSC analyses we found that for samples of enalapril maleate from the compounding pharmacies displayed their $\mathrm{T}_{\text {onset }}$ around $150^{\circ} \mathrm{C}$ and the enthalpy of fusion between $173-213{ }^{\circ} \mathrm{C}$. The X-ray powder diffraction analyses were performed to examine the crystallinity of the samples. Rietveld refinements were performed using the software Topas Academic V4.1 from XRPD data and we verified a good agreement between the calculated and observed XRPD patterns of the samples.

[1] Kiang YH; Hug A; Stephens PW; Xu W, Journal of Pharmaceutical Science. 2003, 92, 1844-1853. 